\title{
AN INVESTIGATION OF WATERMARKING MEDICAL IMAGES
}

\author{
Majdi Al-qdah ${ }^{1}$ and Anas Alqudah ${ }^{2}$ \\ ${ }^{1}$ Department of Computer Engineering, University of Tabuk, Tabuk City, KSA \\ ${ }^{2}$ Department of Finance, College of Business, University of Abu Dhabi, UAE
}

\begin{abstract}
This paper presents the results of watermarking selected various medical cover images with simple string of letters image (patients' medical data) using a combination of the Discrete Wavelet Transform (DWT) Discrete Cosine Transform (DCT) and singular value decomposition (SVD). The visual quality of the watermarked images (before and after attacks) was analyzed using PSNR and four visual quality metrics (WSNR, MSSIM, PSNR-HVS-M, and PSNR-HVS). The PSNR, PSNR-HVS-M, PSNR-HVS, and WSNR average values of the watermarked medical images before attacks were about the $32 \mathrm{db}, 35 \mathrm{db}$, and $42 \mathrm{db}, 40 \mathrm{db}$ respectively; while the MSSM index indicated a similarity of more than $97 \%$ between the original and watermarked images. The metric values decreased significantly after attacking the images with various operations but the watermark image could be retrieved after almost all attacks. Thus, the initial results indicate that watermarking medical images with the patients' data does not significantly affect their visual quality and they still can be utilized for their medical purpose.
\end{abstract}

\section{KEYWORDS}

Watermarking, medical images, DWT, DCT, SVD, visual metrics

\section{INTRODUCTION}

There is an increasing need to store and transfer medical images over computer networks for sharing among doctors. Data hiding has increasingly become an important tool in authentication of images and protection of owners copyright. Image watermarking, which hide important details inside images techniques can be divided into two broad domains: spatial domain and frequency domain $[1,2]$. Three of the most important frequency watermarking methods are the discrete cosine transform (DCT), discrete wavelet transform (DWT) and Singular Value Decomposition (SVD). Various medical images based watermarking schemes have been proposed in literature [3,4,5]. Many researchers have used a hybrid of two or more transforms in order to compensate for the shortcomings of various transforms.

\section{METHODOLOGY}

\subsection{Watermarking algorithms}

This paper uses a combined approach of the discrete wavelet transform (DWT), the discrete cosine transform (DCT), and the singular value decomposition (SVD) watermarking. The DWT decomposes an image into frequency channels of constant bandwidth on a logarithmic scale by separating an image into a set of four non-overlapping multi-resolution sub bands denoted as

Natarajan Meghanathan et al. (Eds) : NLP, JSE, CST, SIP, ARIA - 2018

pp. 87- 95, 2018. (C) CS \& IT-CSCP 2018

DOI : $10.5121 /$ csit.2018.80209 
lower resolution approximation image (LL), horizontal (HL), vertical (LH) and diagonal (HH) with the availability of multiple scale wavelet decomposition. The watermark is usually embedded into the high frequency detail sub-bands (HL, LH and HH sub-band) because the human visual system (HVS) is sensitive to the low-frequency LL part of the image. In general, sensitive data such as medical information are embedded in higher level sub-bands since the detail levels carry most of the energy of the image [6]. Wavelet transform methods achieve higher robustness since they have the characteristics of space frequency localization, multiresolution representation, multi-scale analysis, adaptability and linear complexity [7].

The DCT has a very good energy compaction property. It works by separating the image into different low, high, and middle frequency coefficients [8]. The watermark is embedded in the middle frequency band that gives additional resistance to the lossy compression techniques with less modification of the cover image. The DCT coefficients $D(i, j)$ matrix of an image $(\mathrm{N} x \mathrm{M})$ with pixel intensity $\mathrm{I}(\mathrm{x}, \mathrm{y})$ are obtained as follows:

$$
\begin{aligned}
& D(i, j)=\frac{1}{\sqrt{2 N}} C(i) C(j) \sum_{x=0}^{N-1} \sum_{y=0}^{M-1} I(x, y) \cos ((2 x+1) i \pi / 2 N) \cos ((2 y+1) i \pi / 2 M) \\
& C(i), C(j)=\frac{1}{\sqrt{N}}, \frac{1}{\sqrt{M}} \text { for } i, j=0 \text { and } C(i), C(j)=\sqrt{\frac{2}{N}}, \sqrt{\frac{2}{M}} \text { for } i, j=1,2, \ldots \ldots N-1 \text { or } M-1
\end{aligned}
$$

The (SVD) of a rectangular matrix $\mathrm{R}_{\mathrm{m}}$ is a decomposition of the form

$$
\mathrm{R}_{\mathrm{m}}=\mathrm{USV}^{\mathrm{T}}
$$

Where $\mathrm{R}_{\mathrm{m}}$ is a $\mathrm{M} \times \mathrm{N}$ matrix, $\mathrm{U}$ and $\mathrm{V}$ are orthonormal matrices, and $\mathrm{S}$ is a diagonal matrix comprised of singular values of $\mathrm{R}_{\mathrm{m}}$. The singular values $S_{1} \geq S_{2} \geq S_{3} \geq \ldots \ldots \ldots . S_{n-1} \geq S_{n} \geq 0$ are unique values that appear in descending order along the main diagonal of $S$. They are obtained by taking the square root of the Eigen values of $R_{m} R_{m}^{T}$ and $R_{m}^{T} R_{m}$. The $U, V$ are not unique. In the Singular Value Decomposition, the slight variations of singular values do not affect the visual perception of the cover image, which achieves better quality of the watermarked image and better robustness against attacks. Also, singular values represent the intrinsic algebraic image properties [8].

Various medical images based watermarking schemes have been proposed in literature $[9.10,11]$. Many researchers have used a hybrid of two or more transforms in order to compensate for the shortcomings of various transforms; i.e. in image compression [12], image denoising [13], or image coding [14], and watermarking [15]. In this work, a combined approach of the three transforms is used for watermarking: DWT, DCT, and SVD. The combination of the three transforms increases the robustness and imperceptibility of the watermarked images [16]. Figure 1 shows the approach taken in embedding a watermark (patients data) into a cover image (medical image); The singular values of the watermark (after DCT transformed) are embedded in the singular values of the cover image (after DWT transformed). Figure 2 shows the extraction approach of the patient's image data from the watermarked image. The watermarked images is DWT and DCT transformed then SVD is applied to the DCT coefficients; the watermark is extracted from the LL sub band of DWT. For an added security, the watermark image can be encrypted before embedding it in the cover image, which is not utilized in this paper. 


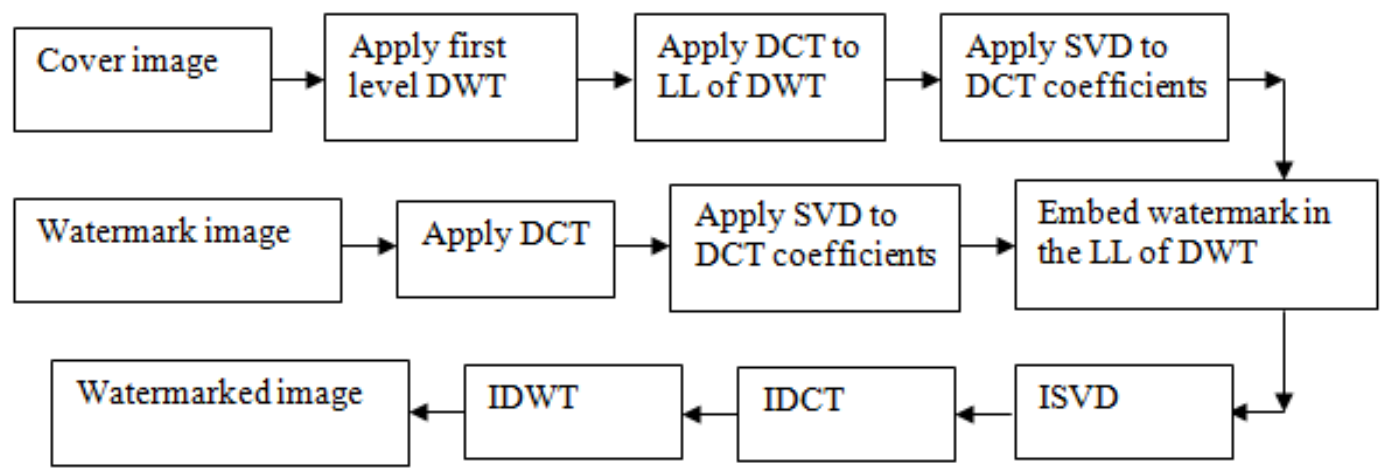

Figure1. Embedding process



Figure 2. Extraction process

\subsection{Performance Measures}

This work utilizes the visual metrics (WSNR, MSSIM, PSNR-HVS-M, and PSNR-HVS) described by Ponomarenko et. al. [17] for comparing the watermarked images with their originals. Traditionally, the efficiency of an image processing operation ; i.e. lossy compression is usually analyzed in terms of rate-distortion curves. These curves represent dependencies of PSNR (or MSE) on bits per pixel (bpp) or compression ratio (CR) where PSNR and MSE are calculated for some original image and the corresponding processed image.

$\operatorname{PSNR}=10 \log \left(\frac{255^{2}}{\mathrm{MSE}}\right)$

MSE $=\sum_{i=1}^{\mathrm{N}} \sum_{\mathrm{j}=1}^{\mathrm{M}} \frac{\left(\mathrm{I}_{\mathrm{ij}}^{\mathrm{d}}-\mathrm{I}_{\mathrm{ij}}^{\mathrm{n}}\right)^{2}}{\mathrm{NM}}$

where ${ }^{I_{i j}^{d j}} I_{i j}^{n}$ denote the values of the original and processed pixels and $N, M$ denote an image size $[18,17]$. In order to obtain a high imperceptibility of the watermarked image, it is desirable to have a high value of PSNR; meaning a lesser value of MSE.

Also, usually the similarity and differences between an original image and a processed image is measured by the Normalized Correlation (NC). Its value is generally 0 to 1 . Ideally it should be 1 but a value 0.7 or higher is usually acceptable [13].

$$
\mathrm{NC}=\sum_{\mathrm{i}=1}^{\mathrm{X}} \sum_{\mathrm{j}=1}^{\mathrm{Y}}\left(\mathrm{I}_{\mathrm{ij}}^{\mathrm{d}} \mathrm{XI} \mathrm{I}_{\mathrm{ij}}^{\mathrm{n}}\right) / \sum_{\mathrm{i}=1}^{\mathrm{X}} \sum_{\mathrm{j}=1}^{\mathrm{Y}}\left(\mathrm{I}_{\mathrm{ij}}^{\mathrm{d}}\right)^{2}
$$

where ${ }^{I_{i j}^{d}} I_{i j}^{n}$ denote the values of the original and processed pixels and $X, Y$ denote an image size.

On the other hand, in evaluating the quality of the watermarked images, it is well known that conventional quality metrics, such as MSE, SNR and PSNR do not always correlate with image 
visual quality [20,21]. In other words, two different distorted images with the same value of PSNR with respect to the same original image, may give significantly different visual impact. Therefore, the choice of a proper visual quality metric for analysis and comparisons is always a problem and can be argued since the human visual system (HVS) is nonlinear and it is very sensitive to contrast changes and to noise [22]. Many studies have confirmed that the HVS is more sensitive to low frequency distortions rather than high frequency components. The best performance was achieved by the metrics PSNR-HVS-M, PSNR-HVS, and WSNR [18] especially if there is noise or the images are to be compressed. HVS-based models are the result of trade-off between computational feasibility and accuracy of the model. HVS-based models can be classified into two categories: neurobiological models and models based on the psychophysical properties of human vision. Psychophysical HVS-based models are implemented in a sequential process that includes luminance masking, colour perception analysis, frequency selection, and contrast sensitivity [22].

More recent ways to evaluate processing of images is by using perceptual image quality assessment methods, which attempt to simulate the functionality of the relevant early human visual system (HVS) components. These methods usually involve a pre-processing process that may include image alignment, point-wise nonlinear transform, low-pass filtering that simulates eye optics, and color space transformation, a channel decomposition process that transforms the image signals into different spatial frequency as well as orientation selective subbands, an error normalization process that weights the error signal in each subband by incorporating the variation of visual sensitivity in different subbands, and the variation of visual error sensitivity caused by intra- or inter-channel neighbouring transform coefficients, and an error pooling process that combines the error signals in different subbands into a single quality/distortion value [23].

PSNR-HVS takes into account the HVS properties such as sensitivity to contrast change and sensitivity to low frequency distortions; while the PSNR-HVSM takes into account the contrast sensitivity function (CSF). Similar to PSNR and MSE, the visual quality metrics PSNR-HVS and PSNR-HVSM can be determined:

$$
\begin{aligned}
& \text { PSNR }- \text { HVS }=10 \log \frac{255^{2}}{\mathrm{MSE}_{\mathrm{nf}}^{\mathrm{HVs}}} \\
& \mathrm{MSE}_{\mathrm{nf}}^{\mathrm{HVS}}=\mathrm{K}^{\left.\sum_{\mathrm{i}=1}^{\mathrm{l}-7} \sum_{\mathrm{j}=1}^{\mathrm{J}-/} \sum_{\mathrm{m}=1}^{\mathrm{s}} \sum_{\mathrm{n}=1}^{\mathrm{g}}\left(\mathrm{X}[\mathrm{m}, \mathrm{n}]_{\mathrm{i}, \mathrm{j}}-\mathrm{X}[\mathrm{m}, \mathrm{n}] \mathrm{ij}_{\mathrm{j}}^{\mathrm{e}}\right) \mathrm{T}_{\mathrm{c}}[\mathrm{m}, \mathrm{n}]\right)}
\end{aligned}
$$

where $\mathrm{I}, \mathrm{J}$ denote image size, $\mathrm{K}=1[(\mathrm{I}-7)(\mathrm{J}-7) 64], \mathrm{X}[\mathrm{m}, \mathrm{n}]_{\mathrm{i}, \mathrm{j}}$ are DCT coefficients of $8 \mathrm{x} 8$ image block for which the coordinates of its left upper corner are equal to i and j, Xij e are the DCT coefficients of the corresponding block in the original image, and $T_{c}[m, n]$ is the matrix of correcting factors [24].

The Weighted Signal to Noise Ratio (WSNR) is a noise metric where the difference (residual) between the original and the processed images must be noise. (WSNR) uses a Contrast Sensitivity Function (CSF) given by the following:

$\operatorname{CSF}=2.6\left(0.02+0.1 f_{a}\right) e^{-\left(0.1 f_{2}\right)^{1.1}}$

where $f_{a i s}$ a radial angular frequency

The WSNR between an original image $(\mathrm{x})$ and a processed image $(\mathrm{y})$ is:

WSNR $=10 \log _{10}\left(\frac{\sum|\operatorname{CSF} \times X(u, v)|^{2}}{\left.\sum \mid \operatorname{CSF} \times X(u, v)-Y(u, v)\right)\left.\right|^{2}}\right)$.

The structural similarity index (SSIM) measures the similarity between two images [19]. SSIM compares two images using information about luminous, contrast and structure. SSIM metric is calculated on various windows of an image. The measure between two windows $\mathrm{x}$ and $\mathrm{y}$ of common size $\mathrm{N} \times \mathrm{N}$ is given as follows: 
$\operatorname{SSIM}(x, y)=\frac{\left\{\left(2 u_{x} u_{y}+\iota_{1}\right)\left(2 \sigma_{x y}+\iota_{2}\right)\right\}}{\left\{\left(u_{x}^{2}+u_{y}^{2}+C_{1}\right)\left(\sigma_{x}^{2}+\sigma_{y}^{2}+C_{2}\right)\right\}}$

SSIM takes values between 1 and $-1 ; \mathrm{u}_{\mathrm{x}}$ is an average of $\mathrm{x}, \mathrm{u}_{\mathrm{y}}$ is an average of $\mathrm{y}, \sigma_{\mathrm{x}}, \sigma_{\mathrm{y}}$ are the standard deviations between the original and watermarked image pixels; while $\mathrm{C}_{1}, \mathrm{C}_{2}$ are positive constant chosen to avoid the instability of SSIM measure.

MSSIM (Multi-Scale Structural Similarity) is a multi-scale extension of a SSIM metric. MSSIM [28] is introduced to incorporate the variations of viewing conditions to the previous single-scale SSIM measure. MSSIM is known as mean structural similarity index metric [25] and it is given by:

$\operatorname{MSSIM}(\mathrm{x}, \mathrm{y})=\frac{1}{\mathrm{M}} \sum_{\mathrm{i}=1}^{\mathrm{M}} \operatorname{SSIM}\left(\mathrm{x}_{\mathrm{i}}, \mathrm{y}_{\mathrm{i}}\right)$

where $\mathrm{M}$ is the correlation between two images $\mathrm{x}, \mathrm{y}$

Correlation is a similarity measure between two functions. The correlation measure between two functions $\mathrm{x}(\mathrm{x}, \mathrm{y})$ and $\mathrm{s}(\mathrm{x}, \mathrm{y})$ in discrete form is defined as:

$\mathrm{M}=\sum_{\mathrm{x}=0}^{\mathrm{M}-1} \sum_{\mathrm{y}=0}^{\mathrm{N}-1} \mathrm{r}(\mathrm{x}, \mathrm{y})[\mathrm{s}(\mathrm{x}, \mathrm{y})]^{*}$

Where []$^{n}$ is the complex conjugate, $\mathrm{x}=0,1, \ldots \ldots ., \mathrm{M}-1$ and $\mathrm{y}=0,1, \ldots \ldots, \mathrm{N}-1$

\section{RESULTS AND DISCUSSIONS}

Five medical cover images of size [512×512] and a watermark image of size [256×256] are selected for analysis shown in Figure 3. The medical cover images contain medical information based on the characteristics of each image and the purpose of its capture. The medical images reveal characteristics of the bones, tissues, vessels, nerves....etc. Thus, embedding a watermark image inside a medical cover image should preserve the existing medical information in the cover medical image: the unique pattern of the fingerprint, vessels and optical nerves inside the retina, bone fracture in the wrist, size and development signs of the fetus, shape, and sliced layers and soft tissue of the human skull. The patients' personal details can be embedded in the captured medical image in textual or image format and saved in one file. The personal details (watermark) are embedded by a combined method of DWT, DCT, and SVD transforms; while the imperceptivity of the watermarked images is evaluated using PSNR, P-HVS, P-HVS-M, WSNR, and MSSIM.

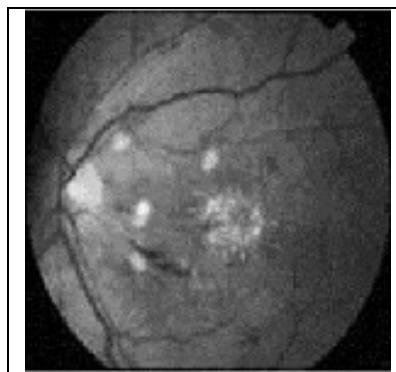

Retina

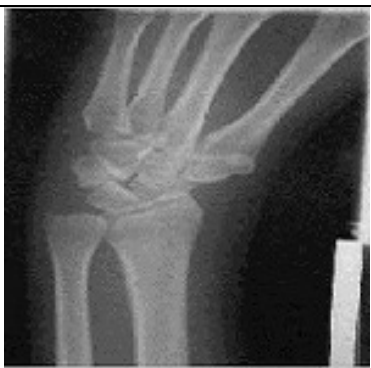

Broken wrist

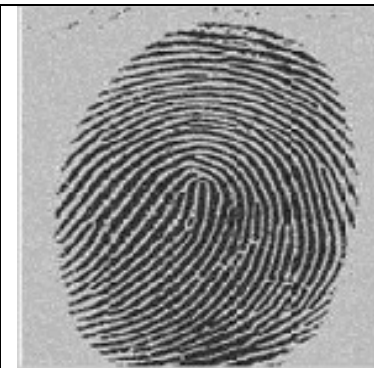

Fingerprint 


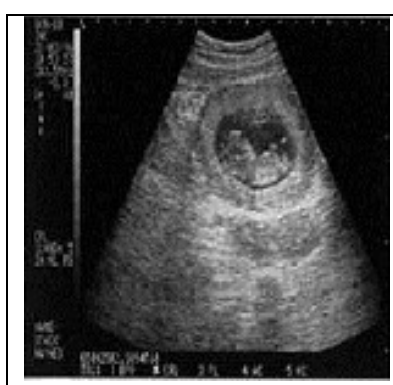

Ultrasound

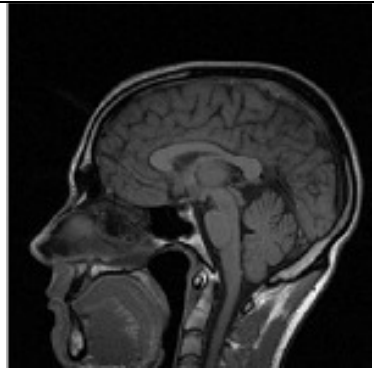

MRIofHead patient name:

patient ID:

Age:

Watermark

Figure 3. Images and Watermark

Table 1 shows the values of PSNR, P-HVS, P-HVS-M, WSNR, and MSSIM metrics of the watermarked images before any attacks. The PSNR average value is about $32 \mathrm{db}, \mathrm{P}-\mathrm{HVS}$ average value is around $35 \mathrm{db}$, P-HVS-M average value is about $42 \mathrm{db}$, and the WSNR average value varies from $35 \mathrm{db}$ to $47 \mathrm{db}$. The MSSIM metric shows that the watermarked images are highly visually similar to the original images with similarity values of more than $0.97 \%$ between the original and the watermarked images. Also, it can be observed that there is no significant difference between the average metric values among the various images; only the WSNR value of the MRIofHead image varies from one image to another with approximately $15 \mathrm{db}$ difference between the Fingerprints image and the MRIofHead image; that is due to the characteristics of the two images.

Table 1. Metric values of the watermarked images

\begin{tabular}{|l|l|l|l|l|l|}
\hline Image & PSNR & P-HVS & P-HVS-M & WSNR & MSSIM \\
\hline Fingerprints & 32.7049 & 34.8745 & 46.2079 & 47.0602 & 0.9920 \\
\hline Retina & 32.9101 & 34.8738 & 40.4924 & 38.0317 & 0.9740 \\
\hline Broken Wrist & 32.7310 & 34.9020 & 40.7815 & 43.3029 & 0.9734 \\
\hline Ultrasound & 33.2059 & 34.8428 & 41.3834 & 37.8052 & 0.9850 \\
\hline MRIofHead & 33.3870 & 35.1103 & 40.0242 & 34.3916 & 0.9770 \\
\hline
\end{tabular}

To test the imperceptivity of the watermarked image, they were attacked with various types of attacks. Tables 2 shows the average values of the same metrics for all the image after the watermarked images are attacked with various operations (Gaussian noise, Salt \& Pepper noise, 2D FIR filter, Cropping, Rotation \& Cropping, Weiner filter, Intensity adjustment, Gaussian filter, and Sharpening). It is shown that the numerical values decrease after an attack operation is performed on the images. Thus, there is a degradation in the quality of the attacked images. The drop in the numerical values is not significant after the Gaussian Noise, Salt \& Pepper Noise, and 2D FIR filter attacks. The values of PSNR, P-HVS, P-HVS-M, and WSNR stay above the value of $20 \mathrm{db}$ and the MSSIM metric values remain above $0.82 \%$. On the other hand, there is a significant decrease in the values after the Cropping, Rotation \& Cropping, Weiner Filter, Intensity adjustment, Gaussian filter, and Sharpening image attack operations. The numerical values of PSNR, P-HVS, P-HVS-M, and WSNR drop to less than $6 \mathrm{db}$ while the MSSIM similarity index drops to $10 \%$ approximately. On the other hand, there is no correlation between the drop in the metric values and the recovery of the watermark; for example, the P-HVS, PHVS-M, and the WSNR values drop greatly after the sharpening attack but the watermark is recovered perfectly. 
Table 2. Average metric values of all five watermarked images after some attacks

\begin{tabular}{|l|l|l|l|l|l|}
\hline Attack & PSNR & P-HVS & P-HVS-M & WSNR & MSSIM \\
\hline No attack & 32.9878 & 34.9207 & 41.7779 & 40.1183 & 0.9803 \\
\hline Gaussian Noise & 19.9103 & 19.9790 & 22.6101 & 27.0916 & 0.8212 \\
\hline Salt\&Pepper Noise & 24.6345 & 24.8935 & 27.9674 & 32.1470 & 0.9304 \\
\hline 2D FIR filter & 25.3646 & 26.6690 & 30.0951 & 35.1960 & 0.9618 \\
\hline Cropping & 13.7011 & 9.5336 & 9.5680 & 8.1108 & 0.7391 \\
\hline Rotation\&Cropping & 5.9136 & 1.7654 & 1.7872 & 0.2728 & 0.0982 \\
\hline Weiner Filter & 5.9212 & 1.7732 & 1.7950 & 0.2801 & 0.1029 \\
\hline Intensity adjustment & 5.9411 & 1.7932 & 1.8150 & 0.3001 & 0.1113 \\
\hline Gaussian filter & 5.9212 & 1.7733 & 1.7950 & 0.2801 & 0.1030 \\
\hline Sharpening & 5.9214 & 1.7733 & 1.7951 & 0.2801 & 0.1031 \\
\hline
\end{tabular}

Finally, this research cannot determine how much of medical information is lost after watermarking medical images or even after attacking the images with a watermark image. Only medical doctors can decide the important segments of a medical image that are affected by watermarking or by attacks; and the effects can vary from one image to another. Also, recovering the watermark after some attacks does not necessarily indicate that all medical information is preserved in the cover image.

\section{CONCLUSiOnS}

The initial results show that watermarking medical images with the patients' personal details does not significantly affect their visual appearance and they can be used by medical staff for their medical purpose if the watermarked images are not attacked; it was experimentally demonstrated that the watermarked medical images appeared similar to their originals and the Human Visual System (HVS) metrics proved a high quality watermarked images. Also, choosing the appropriate watermarking algorithm is essential to obtain the robustness, imperceptivity and security needed to protect the patients' personal data inside a medical image. There are many transform domain algorithms that are available and can be utilized to preserve the characteristics of the original images. A future direction of this research will involve artificial intelligence methods to watermark the images.

\section{ACKNOWLEDGMENT}

The authors would like to acknowledge financial support of this work from the Deanship of Scientific Research (DSR), University of Tabuk, Tabuk, Saudi Arabia, under grant no. S/0180/1438

\section{REFERENCES}

[1] Ashourian (2006), A new mixed spatial domain watermarking of three dimensional triangle mesh, proceeding of the 4th international conference on computer graphics and interactive techniques in Australia and Southeast Asia

[2] Ahmed (2008), Intelligent watermark recovery using spatial domain extension, International conference on intelligent information hiding and multimedia signal processing, IIHMSP' 08 
[3] Lai, C.C., Tsai, C.C. (2010): Digital Image Watermarking Using Discrete Wavelet Transform and Singular Value Decomposition. IEEE Transactions on Instrumentation and Measurement 59(11), 3060-3063

[4] Soliman MM, Hassanien AE, Ghali NI, Onsi HM, (2012) "An Adaptive Watermarking Approach for Medical Imaging using Swarm Intelligence", Int Journal Smart Home 6:37-50

[5] Zain J, Clarke M, (2011) Security in Telemedicine: Issue in Watermarking Medical Images, International Conference: Science of Electronic, Technologies of Information and Telecommunications

[6] Giakoumaki A, Pavlopoulos S, KoutsourisD (2006) Secure and efficient health data management through multiple watermarking on medical images. Med Biol Eng Comput 44: 619-631

[7] Lin W-H, Wang Y-R, Horng S-J, Kao T-W, Pan Y (2009) A blind watermarking method using maximum wavelet coefficient quantization. Expert Syst Appl 36(9) : 11509-11516

[8] Liu, R., Tan, T. (2002): An SVD-based watermarking scheme for protecting rightful ownership, IEEE Transactions on Multimedia 4(1), 121-128

[9] Lai, C.C., Tsai, C.C (2010).: Digital Image Watermarking Using Discrete Wavelet Transform and Singular Value Decomposition. IEEE Transactions on Instrumentation and Measurement 59(11), 3060-3063

[10] Soliman MM, Hassanien AE, Ghali NI, Onsi HM, (2012) "An Adaptive Watermarking Approach for Medical Imaging using Swarm Intelligence", Int Journal Smart Home 6:37-50

[11] Zain J, Clarke M, Security in Telemedicine: Issue in Watermarking Medical Images, International Conference: Science of Electronic, Technologies of Information and Telecommunications

[12] Paul (2006), A novel VLSI architecture for image compression, Eighth IEEE international symposium on multimedia

[13] Shaick (2000), A hybrid transform method for image denoising. 10th European. Signal Processing Conference,

[14] Chappelie (2004)r, image coding with iterated contourlet and wavelet transforms, international conference on image processing,

[15] Ahire, V.K., Kshirsagar, V. (2011): A Digital Watermarking Scheme Based Discrete Wavelet Transform (DWT) and Discrete Cosine Transform (DCT) for Copyright Protection of Digital Images. International Journal of Computer Science and Network Security 11(8), 208-213

[16] Singh AK, Dave M, Mohan A (2014) Hybrid technique for robust and imperceptible image watermarking in DWT- DCT-SVD domain. Natl Acad Sci Lett 37(4):351-358

[17] N. Ponomarenko, V. Lukin, M. Zriakhov, K. Egiazarian, and J. Astola (2006), Estimation of accessible quality in noise image compression, in Proceedings of European Signal Processing Conference (EUSIPCO ’06), pp. 1-4, Florence, Italy.

[18] S. G. Chang, B. Yu, and M. Vetterli, (2000) Adaptive wavelet thresholding for image denoising and compression, IEEE Transactions on Image Processing, vol. 9, no. 9, pp. 1532-1546.

[19] Z. Wang and A. C. Bovik (2006). Modern Image Quality Assessment. Morgan and Claypool Publishing Company, New York 
[20] Z. Wang, A. C. Bovik, H. R. Sheikh (2004), and E. P. Simoncelli, "Image quality assessment: from error visibility to structural similarity," IEEE Transactions on Image Processing, vol. 13, no. 4, pp. $600-612$

[21] Z. Wang and A. C. Bovik (2009), Mean squared error: love it or leave it? A new look at signal fidelity measures, IEEE Signal Processing Magazine, vol. 26, no. 1, pp. 98-117.

[22] N. Ponomarenko, F. Battisti, K. Egiazarian, J. Astola, and V. Lukin (2009), Metrics performance comparison for color image database, in Proceedings of the 4th International Workshop on Video Processing and Quality Metrics, pp. 1-6, Scottsdale, Ariz, USA, CD-ROM.

[23] Zhou Wang1, Eero P. Simoncelli1 and Alan C (2003). Bovik multi-scale structural similarity for image quality assessment. Proceeding of the 37th IEEE Asilomar Conference on Signals, Systems, and Computers, Pacific Grove, CA, Nov. 9-12, 2003.

[24] N. Nill, (1985) A visual model weighted cosine transform for image compression and quality assessment, IEEE Transactions on Communications COM-33, pp. 551-557.

[25] R. F. Zampolo, R. Seara, (2003) A Measure for Perceptual Image Quality Assessment", in Proc. of Int. Conf. on Image Proc., Barcelona, Spain, pp: 433-436, Sept. 